\title{
Application of Concentrated Windings for High-Power Synchronous Wind Generators
}

\author{
V.N. Antipov ${ }^{1, *}$, A.D. Grozov ${ }^{1}$, and A.V. Ivanova ${ }^{1}$ \\ ${ }^{1}$ Institute of Silicate Chemistry RAS, 2, Makarova emb., Saint Petersburg, 199034, Russia
}

\begin{abstract}
The article analyzes the parameters of various configurations concentrated windings for application in high-power geared and direct drive wind generators. It is specified that the concentrated windings advantages over distributed ones can be fully realized by choosing the right values of pole pairs and the slots number. It is shown that the choice of the winding should take into account not only the highest winding factor, but also to the star of slots for the analysis of the harmonic contents of the magnetomotive force (MMF) and electromotive force (EMF), cogging torque and load torque pulsation. Windings with performance conditions $\mathrm{Z}=12+6 k, 2 p=Z \pm 2, k=0,1,2,3, \ldots$ having an odd number of pole pairs are recommended for high-power wind generators.
\end{abstract}

\section{Introduction}

Progress in creating new materials for permanent magnets led to the development of collector and brushless permanent magnets motors from small-power machines for automotive equipment, fan drives, disk drives to larger ones for elevators and low-speed wind generators. As a rule, these machines are equipped with concentrated windings, the coil of which covers a single slot, and the winding can be single- or double-layer. For a balanced three-phase concentrated winding the various combinations of poles and slots are determined by the relation

$$
\frac{Z}{G C D(Z, 2 p)}=3 k
$$

where $Z$ is the number of slots, $2 p$ is the number of poles, and $\operatorname{GCD}(Z, 2 p)$ is the Greatest Common Divisor, $k=1,2,3, \ldots$.

The specific force developed in the magnetic field of a machine with air gap flux density $B_{\delta}$ and current loading $A$ is equal $f_{\sigma}=k_{w} B_{\delta} A$, where $k_{w}$ - winding factor, and is true for both distributed and concentrated windings. Therefore, in terms of creating an electromagnetic torque windings do not have advantages over each other.

The attractiveness of concentrated windings in comparison with classical distributed windings is explained by the simplicity of their design and lower cost. However, the magnetizing force curve of a concentrated winding contains a significant spectrum of harmonics, which limits the windings use only to synchronous machines, which by their nature have a significant air gap.
For synchronous machines the advantages of using these windings are

- the copper losses reduction because of the end winding have the length shortcut;

- reduction of the cogging torque at no load and load torque ripple;

- improved reliability by decreasing roll-to-roll connections.

Many papers have been appeared recently that consider both the theory of these windings, and specific cases of their application.

In [1], the concentrated windings for a direct drive brushless machine with $q=2 / 5$ and $q=2 / 7$ with a different combination of $Z$ and $2 p$ is analyzed, and a comparison with a distributed winding $2 p=16, z=24, q=1.5$ is given. It is shown that increasing the number of slots and poles reduces the cogging torque and the load torque pulsation. A prototype of $10 \mathrm{Nm}, 250 \mathrm{rpm}$ with a concentrated winding $Z=24,2 p=28$ was manufactured and studied. In [2], concentrated windings with $q=2 / 5, q=2 / 7, q=4 / 11$, $q=4 / 13$ are considered for various combinations of $Z$ and $2 p$. A comparison is made with distributed windings in 10 positions, and the advantage of concentrated windings in reducing the cogging torque and the load torque pulsation is shown.

In [3], three options of the machine $(10 \mathrm{~kW}, 400 \mathrm{~V}$, $2000 \mathrm{rpm})$ are investigated: one with a distributed winding $Z=60,2 p=10, q=2$ and two with concentrated windings $Z=12,2 p=10, q=2 / 5$ and $Z=9,2 p=10, q=0.3$. The best option is considered with $q=2 / 5$. Two options of a $55 \mathrm{~kW}, 10000 \mathrm{rpm}$ traction motor with a concentrated winding $Z=36,2 p=30, q=2 / 5$ were studied in [4] under various EMF restrictions at the maximum speed. Article [5] is devoted to single-layer distributed windings, and articles [6-8] contain elements of the concentrated windings theory, and the most complete possible

* Corresponding author: bht@mail.ru 
Table 1. Characteristics of the windings selected for analysis.

\begin{tabular}{|c|c|c|c|c|c|c|c|c|c|}
\hline $\boldsymbol{F}(\mathbf{H z})$ & $\boldsymbol{Z}$ & $\mathbf{2}$ & $\boldsymbol{q}$ & $\boldsymbol{q}_{\boldsymbol{n}}$ & $\boldsymbol{q}_{\boldsymbol{d}}$ & $\boldsymbol{s e g}$ & $\mathbf{L C M}=\boldsymbol{t}$ & $\boldsymbol{Z} / \boldsymbol{m} / \boldsymbol{t}$ & $\boldsymbol{Z} / \boldsymbol{t}$ \\
\hline \multicolumn{8}{|c|}{ Geared $(n=150 \mathrm{rpm})$} \\
\hline 50 & 48 & 40 & 0.400 & 2 & 5 & 4 & 4 & 4 & 12 \\
\hline 52.5 & 54 & 42 & 0.429 & 3 & 7 & 3 & 3 & 6 & 18 \\
\hline 50 & $42 *$ & 40 & 0.350 & 7 & 20 & $\mathrm{k}=5$ & 2 & 7 & 21 \\
\hline \multicolumn{8}{|c|}{ Ddirect-drive $(n=10 \mathrm{rpm})$} \\
\hline 10 & 144 & 120 & 0.4 & 2 & 5 & 12 & 12 & 4 & 12 \\
\hline 10.5 & 162 & 126 & 0.429 & 3 & 7 & 9 & 9 & 6 & 18 \\
\hline 10.17 & $120^{*}$ & 122 & 0.327 & 20 & 61 & $\mathrm{k}=18$ & 1 & 40 & 120 \\
\hline 9.83 & $120^{*}$ & 118 & 0.339 & 20 & 59 & $\mathrm{k}=18$ & 1 & 40 & 120 \\
\hline
\end{tabular}

spectrum of combinations of $Z$ and $2 p$ is presented in [7], for which the winding coefficients are determined and it is noted that some versions cause increased vibration and noise.

Finnish researchers of high power wind generators [9] proposed an option of $8 \mathrm{MW}$ direct-drive generator with a concentrated winding $Z=144,2 p=120, q=2 / 5$ (12 segments, $Z=12,2 p=10)$. The authors also have considered options of high-power generators with concentrated windings [10]. It has been proposed to give preference to the choice of a concentrated winding with tangential magnetization, in which for generators with a rotation speed of $150 \mathrm{rpm}$ and $12 \mathrm{rpm}$, the efficiency is higher and the active materials mass is less.

The above analysis of the properties of concentrated windings shows that the choice of the winding should take into account not only the highest winding factor, but also to analyze the star of slots, harmonic composition of magnetomotive force, harmonic spectrum EMF, cogging torque and load torque ripple, as well as emerging losses.

\section{Selecting the research object}

In the last decade, a significant part of international research has focused on creating a wind turbine with a direct-drive synchronous generator [9-14], but the main market is still occupied by generators with a single-stage gearbox [7]. In this regard, generators with nominal rotation speeds $n=150 \mathrm{rpm}$ and $n=10 \mathrm{rpm}$ were selected for analysis. Based on the established practice of wind turbine construction, geared generators with a rotation speed of $n=150 \mathrm{rpm}$ are designed for the $50 \mathrm{~Hz}$ frequency of alternating current, and gearless generators for a reduced frequency of $10 \mathrm{~Hz}$. The windings selected for analysis are shown in Table 1.

The selected windings can be divided into two groups. The first group has a constant value of the number of slots per pole and phase $q=q_{n} / q_{d}$ regardless of the simultaneous increase in the number of slots and the number of pole pairs. Depending on the required speed and $\mathrm{AC}$ frequency, the winding has a repeating base fragment, for example, when $q=2 / 5$, the base fragment $Z=12,2 p=10$. In the table, the number of repetitions is given in the seg column. The second group of windings meets the conditions of execution $Z=12+6 k, 2 p=Z \pm 2$, $k=0,1,2, \ldots$ and it has a different arrangement structure for an even and odd number of pole pairs $p$ and a different value for the number of slots per pole and phase $q$ when $k$ changes. These windings are indicated in the table by *. The periodicity of the winding is determined by the Greatest Common Divisor of the numbers of slots and pole pairs $t=\operatorname{GCD}(Z, p)$. All the windings shown in the table are balanced, since they satisfy condition (1). The star of the groove EMF of each winding has $Z / t$ arrows, each of which has $t$ vectors. The angle between the vectors of two adjacent slots in electrical degrees is equal to $\alpha_{s}^{e}=p \alpha_{s}^{m}$, where $\alpha_{s}^{m}=2 \pi / Z$ is the groove angle in mechanical radians, the resulting angle between two arrows $\alpha_{p h}=\frac{\alpha_{s}^{e}}{p} t$.

\section{Winding factor}

The winding factor of concentrated winding is defined as for the distributed one in the form of the product of the distribution and the reduction coefficients $k_{w}=k_{d} k_{s}$.

The formula for calculating the distribution and reduction coefficients has the same structure as the formula for the distributed winding:

$$
\begin{gathered}
k_{d}=\frac{\sin \left(\frac{q_{p h}}{2} \frac{\alpha_{p h}}{2}\right)}{q_{p h} \sin \left(\frac{\alpha_{p h}}{4}\right)}, \\
k_{s}=\sin \left(\frac{\beta \pi}{2}\right)=\cos \left(\frac{c_{a}}{q} \frac{\pi}{2 m}\right),
\end{gathered}
$$

So in [5] the following expressions are proposed: - distribution coefficient for even and odd $q_{p h}$ 
Table 2. Evaluation of the winding coefficient and the tooth component of the torque.

\begin{tabular}{|c|c|c|c|c|c|c|c|c|}
\hline $\boldsymbol{Z} / 2 \boldsymbol{p}$ & $\boldsymbol{q}_{\boldsymbol{n}} / \boldsymbol{q}_{\boldsymbol{d}}$ & $\boldsymbol{q}_{\boldsymbol{p h}}$ & $\boldsymbol{\alpha}_{\boldsymbol{p h}}$ & $\boldsymbol{k}_{\boldsymbol{w}}$ & $\begin{array}{c}\text { LCM } \\
(\boldsymbol{Z}, \boldsymbol{p})\end{array}$ & $\begin{array}{c}\text { LCM } \\
(\boldsymbol{Z}, \mathbf{p} \boldsymbol{p})\end{array}$ & $\boldsymbol{F}$ & $\boldsymbol{\alpha}$ \\
\hline \multicolumn{8}{|c|}{ Geared $(n=150 \mathrm{rpm})$} \\
\hline $48 / 40$ & $2 / 5$ & 2 & 0.131 & 0.933 & 240 & 240 & 5 & $0.833 ; 0.667$ \\
\hline $45 / 42$ & $5 / 14$ & 5 & 0.140 & 0.961 & 315 & 630 & 7 & $0.933 ; 0.867 ; 0.800$ \\
\hline $42 / 40$ & $7 / 20$ & 7 & 0.150 & 0.953 & 420 & 840 & 10 & $0.952 ; 0.905 ; 0.857$ \\
\hline \multicolumn{8}{|c|}{ Direct-drive $(n=10 \mathrm{rpm})$} \\
\hline $144 / 120$ & $2 / 5$ & 2 & 0.044 & 0.933 & 720 & 720 & 5 & $0.833 ; 0.667$ \\
\hline $162 / 126$ & $3 / 7$ & 9 & 0.039 & 0.902 & 1134 & 1134 & 7 & $0.889 ; 0.778 ; 0.667$ \\
\hline $120 / 122$ & $20 / 61$ & 20 & 0.052 & 0.954 & 7320 & 7320 & 61 & $0.983 ; 0.967 ; 0.833$ \\
\hline $120 / 118$ & $20 / 59$ & 20 & 0.052 & 0.954 & 7080 & 7080 & 59 & $0.983 ; 0.967 ; 0.833$ \\
\hline
\end{tabular}

$$
\begin{gathered}
q_{p h}=Z / m t, k_{d}=\frac{\sin \left(\frac{q_{p h}}{2} \frac{\alpha_{p h}}{2}\right)}{\frac{q_{p h}}{2} \sin \left(\frac{\alpha_{p h}}{2}\right)}, \\
k_{d}=\frac{\sin \left(\frac{q_{p h}}{2} \frac{\alpha_{p h}}{2}\right)}{q_{p h} \sin \left(\frac{\alpha_{p h}}{4}\right)}
\end{gathered}
$$

- reduction coefficient

$$
k_{s}=\sin \frac{2 \pi p y_{q}}{2 Z}, y_{q}=\operatorname{round}\{Z /(2 p)\}
$$

Convenient formulas for determining the winding coefficient are presented in [8] for three-phase winding:

$$
k_{d}=\frac{\sin \left(\frac{\pi}{6}\right)}{q \sin \left(\frac{1}{q} \frac{\pi}{6}\right)}, k_{s}=\cos \left(\frac{N_{s p}}{N_{d s f}} \frac{\pi}{6}\right)
$$

The authors propose to calculate the groove and coil cycles for the winding $N_{t c} ; N_{c c}$ for phase $N_{t c p h} ; N_{c c p h}$ and for magnets $N_{m c}$, moreover $N_{t c}=m N_{t c p h} ; N_{c c}=m N_{c c p h}$. Fulfilling the condition $N_{m c}=N_{t c} \pm 1$ provides a high value of the winding coefficient. Low harmonic distortion corresponds to the offset condition between the winding layers $N_{t s}$ which are close to $N_{c c p h} / 2$.

The above formulas are presented for the winding coefficients for the first harmonic. They can also be used to calculate higher harmonics, whose order $v$ should be entered under the sign of $\sin$.

The calculated values of the winding coefficients for the selected windings are shown in Table 2.

All the selected windings have a high value of the winding factor. The lowest value has the winding with $q=3 / 7$.

The use of concentrated windings significantly reduces the tooth cogging torque which is caused by the sum of the interactions of the edge of the magnet with

the opening of the groove. An estimate can be made based on the number of such cycles [2]

$$
F=\frac{\operatorname{LSM}(Z, p)}{Z}
$$

where $\operatorname{LCM}(Z, p)$ is the least common multiple. The higher the $F$, the smaller the cogging torque. The calculated $F$ values are shown in Table 2 and they are significant values for windings of direct-drive generators. For example, a distributed winding, $2 p=16$, $z=24$ has the LCM value is 24 , and $F=1$.

In [15] it is shown that the minimum of the cogging torque value corresponds to the ratio of the pole arc to the pole pitch equal to the one obtained by the expression:

$$
\alpha=1-\frac{2 p k}{\operatorname{LCM}(Z, 2 p)}
$$

Table 2 shows the optimal values of the ratio of the pole arc to the pole pitch for each winding under consideration. Hence, during the design process, it is possible to avoid the harmful influence of the tooth component of the torque. It is especially easy for a gearless drive.

The tooth component of the torque is calculated as a partial derivative of the energy stored in the air gap at the angle of the rotor rotation [6].

\section{Magnetomotive force (MMF) harmonics}

Analysis of the (MMF) distribution curve of the winding is of interest, since the harmonic components contained in it cause additional losses in the stator iron. When calculating harmonics, the winding frequency is important. Harmonics of the order $v=2 p /$ create a positive torque. Fig. 1 shows the MMF distribution in the slots for the winding $Z=48,2 p=40 q=2 / 5$. For this winding, the harmonic that creates the useful torque $v=10$.

Fig. 2 shows the MMF spectra for the windings under consideration obtained by a fast Fourier transform (FFT). 


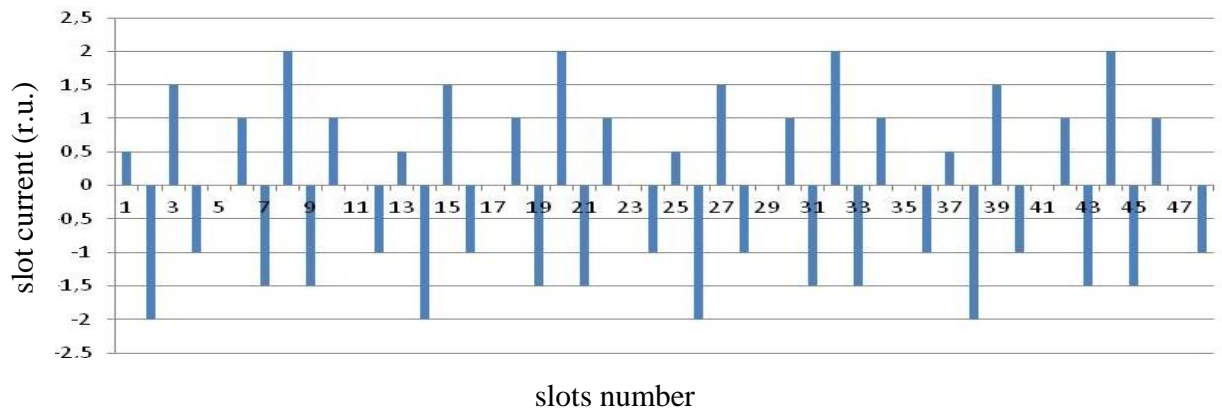

Fig. 1. MMF distribution at the slots for the winding $Z=48,2 p=40, q=2 / 5$.

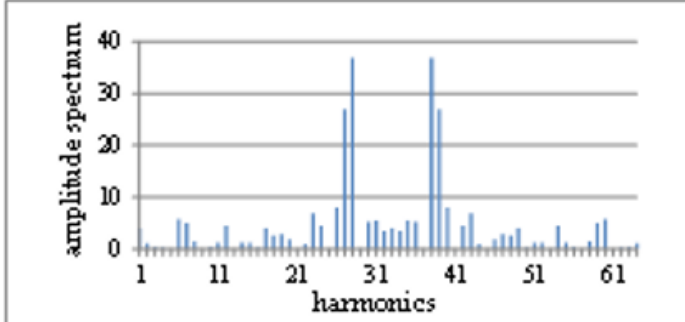

(a)

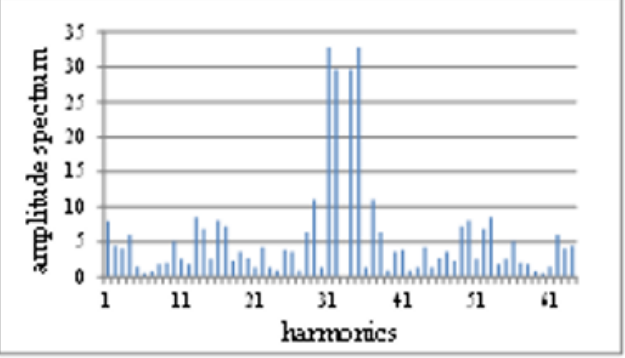

(c)

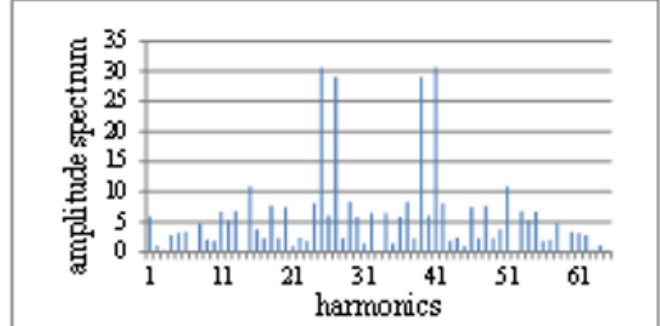

(b)

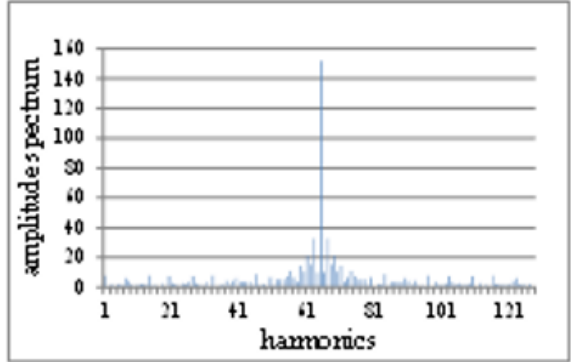

(d)

Fig. 2. MMF Spectra obtained by FFT for windings: a) $q=2 / 5$; b) $q=3 / 7$; $c$ ) $q=7 / 20$; $d$ ) $q=20 / 59$.

Comparison of the spectra shows the advantage of the winding to the conditions of execution $Z=12+6$, $2 p=Z \pm 2, k=0,1,2,3, \ldots$ with an odd number of pole pairs.

\section{The EMF harmonic composition at no load}

The EMF harmonic composition at no load

$$
B_{\text {mag }}\left(\theta_{s}, \theta_{r}, R_{r}\right)=\sum_{n=1,3,5 \ldots}^{\infty} B_{n}\left(R_{r}\right) \cos n p\left(\theta_{s}-\theta_{r}\right)
$$

where $B_{n}$ is the $n^{\text {th }}$ harmonic component, $R_{r}$ is the radius of the air gap [m], and $\theta_{s}$ is the angle along the stator periphery [mech. rad], $\theta_{r}-$ angle of the rotor [mech. rad].

Account for the toothiness either by reducing the flow coupling using the Carter coefficient, or by using a special function $\lambda_{a g}\left(\theta_{s}, R_{r}\right)$ which takes into account the change in flow coupling in both the gap and the magnet and can be represented by a Fourier series

$$
\lambda_{a g}\left(\theta_{s}, R_{r}\right)=\sum_{n=1,3,5 \ldots}^{\infty} \lambda_{n}\left(R_{r}\right) \cos n Z \theta_{s} .
$$

Then the air gap flux density

$$
B_{\delta 0}\left(\theta_{s}, \theta_{r}, R_{r}\right)=\lambda_{a g}\left(\theta_{s}, R_{r}\right) B_{\text {mag }}\left(\theta_{s}, \theta_{r}, R_{r}\right) .
$$

In [10], the results of numerical calculation by FEA of a two-dimensional electromagnetic field of a generator $8000 \mathrm{~kW}, 6300 \mathrm{~V}, 150 \mathrm{rpm}$ with tangential magnetization and a concentrated winding $Z=42,2 p=40$ are presented. There are the $7^{\text {th }}$ harmonic component in the air gap flux density.

\section{Electromagnetic torque and its pulsation}

For concentrated windings with a large number of pole pairs $p$ and small values $q$, the electromagnetic torque is inversely proportional to the synchronous inductance along the longitudinal axis $M_{e l} \propto 1 / p L_{d}$, and since $L_{d} \propto 1 / p q$, the larger torqueis realized at large values of the number of slots per pole and phase $q$. Among the compared windings, the largest electromagnetic torque 
can be realized in the winding with $q=3 / 7$, but this winding has the smallest winding coefficient.

The presence of higher harmonics in phase currents and phase EMF curves causes the load torque pulsations, the instantaneous value of which can be represented as

$$
M(t)=\frac{i_{a}(t) e_{a}(t)+i_{b}(t) e_{b}(t)+i_{c}(t) e_{c}(t)}{\omega_{r}},
$$

here $i(t)$ and $e(t)$ are instantaneous values of phase currents and EMF.

The interaction of the $5^{\text {th }}$ harmonic of the EMF with the main harmonic of the current leads to the appearance of the $4^{\text {th }}$ and 6 th even harmonics in the torque curve. A significant reduction of the $5^{\text {th }}$ harmonic EMF can be achieved by choosing the number of slots $Z$ and pole pairs $p$, the shape of the magnet and saturation. Ripple is also minimized if the opening width of the slot is equal to the width of the tooth. The optimal condition is provided if $Z$ and $2 p$ do not have a common divisor or have a large LCM (the least common multiple) value. Among the windings being compared, the windings for direct drive have the lowest torque ripple, first of all the windings with performance conditions $Z=12+6 k$, $2 p=Z \pm 2$.

The torque ripple for machines with concentrated windings is usually located in the range of 1 to $5 \%$ of the rated torque and in any case it is less than in machines with distributed windings.

\section{Conclusions}

The parameters of various configurations concentrated windings were analyzed for application in high-power gear and gearless wind generators. It is shown that the advantages of concentrated windings over distributed ones can be fully realized by choosing the values of the number of slots and poles.

When designing high-power wind generators, we can recommend to use the concentrated windings with conditions of performance $Z=12+6 k, \quad 2 p=Z \pm 2$, $k=0,1,2,3, \ldots$ having an odd number of pole pairs. Such windings have a high winding coefficient, low presence of higher harmonic in the current and EMF curves, almost negligible cogging torque and low load torque pulsation. Such application is especially effective for direct-drive high-power wind generators.

\section{References}

[1] R. Wrobel, P.H. Mellor, IEEE Trans. on Energy Conversion 23, 1, 1-8 (2008)

[2] R.K. Reichert, PM-motors with concentrated non overlapping windings. Some characteristics, in XVI International Conference on Electrical Machines2004, ICEM Proceedings, Cracow, Poland, 541 (2004)

[3] R. Nuscheler, Comparison of permanent magnet synchronous machines with distributed and concentrated windings, in XVII Intern. Conf. on
Electrical Machines-2006, ICEM Proc., Chania, Greece, 609 (2006)

[4] A.M. El-Refaie, T.M. Jahnsand, J.W. McKeever, Effect of back-EMF constraints on fractional-slot surface PM machines designed for wide constantpower speed range operation, in XVII Inter. Conf. on Electrical Machines-2006, ICEM Proceedings, Chania, Greece, 140 (2006)

[5] N. Bianchi, M. Dai Pre, IEEE Proc. Electr. Power Appl 153, 3, 459-466 (2006)

[6] A.M. El-Refaie, T.M. Jahns, D.W. Novotny, IEEE Trans. on Energy conversion 21, 1, 34-43 (2006)

[7] F. Libert, J. Soulard, Investigation on pole slot combination for permanent magnet machines with concentrated windings, in XVI Intern. Conf. on Electr. Machines-2004, ICEM Proc., Cracow, Poland, 5-8 (2004)

[8] A.D. Gerlando, R. Perini, M. Ubaldini, High pole number, PM synchronous motor with concentrated coil armature windings, in XVI Intern. Conf.e on Electr. Machines-2004, ICEM Proc., Cracow, Poland, 58 (2004)

[9] Y. Alexandrova, R.S. Semken, J. Pyrhonen, Int. Review of Elect. Eng. (IREE) 8, 6, 1728-1737 (2013)

[10] V. Antipov, A. Grozov, A. Ivanova, Electrical Technology Russia 4, 56-63 (2020)

[11] D. Bang, H. Polinder, G. Shrestha, J.A. Ferreira, EPE Journal 18, 3, 7-13 (2008)

[12] J. Zhang, Z. Chen, M. Cheng, IET Renewable Power Generation 1, 4, 203-210 (2007)

[13] H. Polinder, F.A. van der Pijl, IEEE Trans. on Energy Conversion 21, 3, 725-733 (2006)

[14] L. Sethuraman, M. Maness, K. Dykes, Optimized Generator Designs for the DTU 10-MW Offshore Wind Turbine using Generator SE [Electronic resource], in AIAA SciTech Forum: 35th Wind Energy Symp., Grapevine, Texas DOI: 10.2172/1395455 Available at: http://www.theswitch.com/wind-power/permanentmagnet-generators.

[15] Z.Q. Zhu, S. Ruangsinchaiwanich, N. Schofield, D. Howe, IEEE Trans. on Magnetics 39, 5, 32383240 (2003)

[16] E. Ahmed, A. S. Alshebami, and A. G. Polyakova, Renewable Energy use and its Effects on Environment and Economic Growth: Evidence from Malaysia, International Journal of Energy Economics and Policy 10 (5), 50-57 (2020). doi:10.32479/ijeep.10219 